\title{
On the sizes of bipartite 1-planar graphs
}

\author{
Yuanqiu Huang* \\ Department of Mathematics \\ Hunan Normal University \\ Changsha 410081, P. R. China \\ hyqq@hunnu.edu.cn
}

\author{
Zhangdong Ouyang $^{\dagger}$
}

Department of Mathematics

Hunan First Normal University

Changsha 410205, P. R. China

oymath@163.com

\author{
Fengming Dong \\ National Institute of Education \\ Nanyang Technological University \\ Singapore \\ fengming.dong@nie.edu.sg
}

Submitted: Nov 12, 2020; Accepted: Apr 27, 2021; Published: May 21, 2021

(C) The authors. Released under the CC BY-ND license (International 4.0).

\begin{abstract}
A graph is called 1-planar if it admits a drawing in the plane such that each edge is crossed at most once. Let $G$ be a bipartite 1-planar graph with $n(n \geqslant 4)$ vertices and $m$ edges. Karpov showed that $m \leqslant 3 n-8$ holds for even $n \geqslant 8$ and $m \leqslant 3 n-9$ holds for odd $n \geqslant 7$. Czap, Przybyło and Škrabul'áková proved that if the partite sets of $G$ are of sizes $x$ and $y$, then $m \leqslant 2 n+6 x-12$ holds for $2 \leqslant x \leqslant y$, and conjectured that $m \leqslant 2 n+4 x-12$ holds for $x \geqslant 3$ and $y \geqslant 6 x-12$. In this paper, we settle their conjecture and our result is even under a weaker condition $2 \leqslant x \leqslant y$.
\end{abstract}

Mathematics Subject Classifications: 05C10, 05C62

\section{Introduction}

A drawing of a graph $G=(V, E)$ is a mapping $D$ that assigns to each vertex in $V$ a distinct point in the plane and to each edge $u v$ in $E$ a continuous arc connecting $D(u)$ and $D(v)$. We often make no distinction between a graph-theoretical object (such as a vertex, or an edge) and its drawing. All drawings considered here are such ones that no

*Supported by MOE-LCSM, School of Mathematics and Statistics, Hunan Normal University, Changsha, Hunan 410081, P. R. China.

${ }^{\dagger}$ Corresponding author 
edge crosses itself, no two edges cross more than once, and no two edges incident with the same vertex cross. The crossing number of a graph $G$ is the smallest number of crossings in any drawing of $G$.

A drawing of a graph is 1-planar if each of its edges is crossed at most once. If a graph has a 1-planar drawing, then it is 1-planar. The notion of 1-planarity was introduced in 1965 by Ringel [10], and since then many properties of 1-planar graphs have been studied (e.g. see the survey paper [8]).

It is well-known that any simple planar graph with $n(n \geqslant 3)$ vertices has at most $3 n-6$ edges, and a simple and bipartite graph with $n(n \geqslant 3)$ vertices has at most $2 n-4$ edges. Determining the maximum number of edges in 1-planar graphs with a fixed number of vertices has aroused great interest of many authors (see, for example, [2, 4, 6, 9, 13]). It is known that $[2,6,9]$ any 1 -planar graph with $n(n \geqslant 3)$ vertices has at most $4 n-8$ edges. For bipartite 1-planar graphs, an analogous result was due to Karpov [7].

For any graph $G$, let $V(G)$ and $E(G)$ denote its vertex set and edge set.

Theorem 1 ([7]). Let $G$ be a bipartite 1-planar graph. Then $|E(G)| \leqslant 3|V(G)|-8$ when $|V(G)|$ is even and $|V(G)| \neq 6$, and $|E(G)| \leqslant 3|V(G)|-9$ when either $|V(G)|$ is odd or $|V(G)|=6$. For $|V(G)| \geqslant 4$, these bounds are tight.

Note that Karpov's upper bound on the size of a bipartite 1-planar graph is in terms of its vertex number. When the sizes of partite sets in a bipartite 1-planar graph are taken into account, Czap, Przybyło and Škrabul'áková [5] obtained another upper bound for its size (i.e., Corollary 2 in [5]).

Theorem 2 ([5]). If $G$ is a bipartite 1-planar graph with partite sets of sizes $x$ and $y$, where $2 \leqslant x \leqslant y$, then $|E(G)| \leqslant 2|V(G)|+6 x-16$.

For each pair of integers $x$ and $y$ with $x \geqslant 3$ and $y \geqslant 6 x-12$, the authors in [5] constructed a bipartite 1-planar graph $G$ with partite sets of sizes $x$ and $y$ such that $|E(G)|=2|V(G)|+4 x-12$ holds. Moreover, they believed this lower bound is optimal for such graphs and thus posed the following conjecture.

Conjecture $3([5])$. For any integers $x$ and $y$ with $x \geqslant 3$ and $y \geqslant 6 x-12$, if $G$ is a bipartite 1-planar graph with partite sets of sizes $x$ and $y$, then $|E(G)| \leqslant 2|V(G)|+4 x-12$.

In this paper we obtain the following result which proves Conjecture 3.

Theorem 4. Let $G$ be a bipartite 1-planar graph with partite sets of sizes $x$ and $y$, where $2 \leqslant x \leqslant y$. Then $|E(G)| \leqslant 2|V(G)|+4 x-12$.

Note that when $2 \leqslant x \leqslant y \leqslant 6,2|V(G)|+4 x-12 \geqslant x y$ holds. Thus, Theorem 4 is trivial when $2 \leqslant x \leqslant y \leqslant 6$. The result in [5, Lemma 4] shows that the upper bound for $|E(G)|$ in Theorem 4 is tight when $x \geqslant 3$ and $y \geqslant 6 x-12$. Also, if $x<\frac{1}{3}(y+4)$, Theorem 4 provides a better upper bound for $|E(G)|$ than Theorem 1.

The authors in [5] mentioned a question of Sopena [11]: How many edges we have to remove from the complete bipartite graph with given sizes of the partite sets to obtain a 
1-planar graph? By Theorem 4, any bipartite 1-planar graph $G$ with partite sets of sizes $x$ and $y$ can be obtained from the complete bipartite graph $K_{x, y}$ by removing at least the following number of edges

$$
x y-(2(x+y)+4 x-12)=x y-6 x-2 y+12=(x-2)(y-6) .
$$

The next conclusion then follows immediately.

Corollary 5. Let $K_{x, y}$ be the complete bipartite graph with partite sets of sizes $x$ and $y$, where $x \geqslant 2$ and $y \geqslant \min \{6, x\}$. Then at least $(x-2)(y-6)$ edges must be removed from $K_{x, y}$ such that the resulting graph becomes possibly a 1-planar graph, and the lower bound on the number of removed edges is best possible.

The remainder of this paper is arranged as follows. In Section 2, we explain some terminology and notation used in this paper. In Section 3, under some restrictions, we present several structural properties on an extension of $D^{\times}$for a 1-planar drawing $D$ of a bipartite 1-planar graph $G$, where $D^{\times}$is a plane graph introduced in Section 2. Some important lemmas for proving Theorem 4 are given in Section 4, while the proof of this theorem is completed in Section 5. Finally, we give some further problems in Section 6 .

\section{Terminology and notation}

All graphs considered here are simple, finite and undirected, unless otherwise stated. For terminology and notation not defined here, we refer to [1]. For any graph $G$ and $A \subseteq V(G)$, let $G[A]$ denote the subgraph of $G$ with vertex set $A$ and edge set $\{e \in E(G)$ : $e$ joins two vertices in $A\} . G[A]$ is called the subgraph of $G$ induced by $A$. For a proper subset $A$ of $V(G)$, let $G-A$ denote the subgraph $G[V(G) \backslash A]$.

A walk in a graph $G$ is an alternating sequence of vertices and edges, denoted by $W$ :

$$
W: v_{0}, e_{1}, v_{1}, e_{2}, v_{2}, \cdots, v_{k-1}, e_{k}, v_{k}
$$

where $v_{0}$ and $v_{k}$ are the initial vertex and the terminal vertex of the walk, and $e_{i}$ is an edge joining vertices $v_{i-1}$ and $v_{i}$ for all $i=1,2, \cdots, k$; the walk $W$ is closed if its initial vertex $v_{0}$ and terminal vertex $v_{k}$ are the same; the walk (resp., a closed walk) $W$ is a trail (resp., a circuit) if all edges in $W$ are distinct; and a trail (resp., a circuit) $W$ is a path (resp., a cycle) if all vertices in $W$ are also distinct.

The length of a path (resp., cycle) is the number of edges contained in it. A path (resp., a cycle) of length $k$ is said to be a $k$-path (resp., $k$-cycle). If a cycle $C$ is composed of two paths $P_{1}$ and $P_{2}$ which have the same end vertices but have different interior vertices, we sometimes write $C=P_{1} \cup P_{2}$.

A plane graph is a planar graph together with a drawing without crossings, and at this time we say that $G$ is embedded in the plane. A plane graph $G$ partitions the plane into a number of connected regions, each of which is called a face of $G$. If a face is homeomorphic to an open disk, then it is called cellular; otherwise, noncellular. Actually, a noncellular face is homeomorphic to an open disk with a few removed "holes". For a cellular face $f$, 
the boundary of $f$ can be regarded as a closed walk of $G$, while for a noncellular face $f$, its boundary consists of many disjoint closed walks of $G$. The size of a face is the number of the edges contained in its boundary with each repeated edge counted twice. A face of size $k$ is also said to be a $k$-face.

It is known that a plane graph $G$ has no noncellular faces if and only if $G$ is connected. For a connected plane graph $G$, the well-known Euler's formula states that $|V(G)|$ $|E(G)|+|F(G)|=2$, where $F(G)$ denotes the face set of $G$.

A cycle $C$ of a plane graph $G$ partitions the plane into two open regions, the bounded one (i.e., the interior of $C$ ) and the unbounded one (i.e., the exterior of $C$ ). We denote by $\operatorname{int}(C)$ and $\operatorname{ext}(C)$ the interior and exterior of $C$, respectively, and their closures by $I N T(C)$ and $\operatorname{EXT}(C)$. Clearly, $I N T(C) \cap E X T(C)=C$. A cycle $C$ of a plane graph $G$ is said to be separating if both $\operatorname{int}(C)$ and $\operatorname{ext}(C)$ contain at least one vertex of $G$.

Let $D$ be a 1-planar drawing of a graph $G$. The associated plane graph $D^{\times}$is the plane graph that is obtained from $D$ by turning all crossings of $D$ into new vertices of degree four; these new vertices of degree four are called the crossing vertices of $D^{\times}$.

\section{An extension of $D^{\times}$}

Throughout this section, we always assume that the considered graph $G$ (possibly disconnected) is a bipartite 1-planar graph with partite sets $X$ and $Y$, where $3 \leqslant|X| \leqslant|Y|$. Let $D$ be a 1-planar drawing of $G$ with the minimum number of crossings, and $D^{\times}$be the associated graph of $D$ with the crossing vertex set $W$.

Note that subsets $X, Y$ and $W$ form a partition of $V\left(D^{\times}\right)$. We color the vertices in $X, Y$ and $W$ by the black color, white color and red color respectively. $D^{\times}$can be extended to a plane graph, denoted by $D_{W}^{\times}$, by adding edges joining black vertices as described below:

for each vertex $w$ in $W$, that is adjacent to two black vertices in $D^{\times}$, say $x_{1}$ and $x_{2}$, we draw an edge, denoted by $e_{w}$, joining $x_{1}$ and $x_{2}$ which is the "most near" to one side of the path $x_{1} w x_{2}$ of $D^{\times}$such that it does not cross with any other edge, as shown in Figure 1 (b).

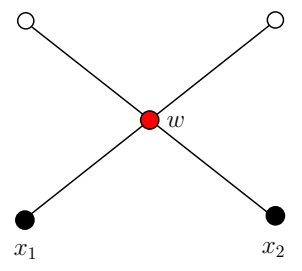

(a)

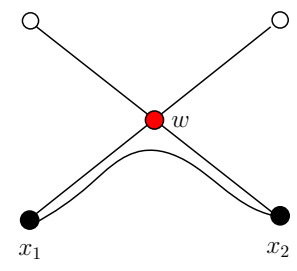

(b)

Figure 1: The extension of $D^{\times}$.

Observe that $D_{W}^{\times}$is a plane graph with $D^{\times}$as its spanning subgraph and the edge set of $D_{W}^{\times}$is the union of $E\left(D^{\times}\right)$and $\left\{e_{w}: w \in W\right\}$. Although $D^{\times}$is a simple graph, $D_{W}^{\times}$ 
might contain parallel edges (i.e., edges with the same pair of ends), as there may exist two edges in $\left\{e_{w}: w \in W\right\}$ with the same pair of ends. An example is shown in Figure 2 (c), where $D$ is a 1-planar drawing of $K_{3,6}$.

Let $F_{D}$ (or simply $F$ ) and $H_{D}$ (or simply $H$ ) denote the subgraphs $D_{W}^{\times}[W \cup X]$ and $D_{W}^{\times}[X]$ respectively. Obviously, $H$ is a subgraph of $F$ and its edge set is $\left\{e_{w}: w \in W\right\}$, while the edge set of $F$ is the union of $E(H)$ and $\left\{w x_{1}, w x_{2} \in E\left(D^{\times}\right): w \in W \& x_{1}, x_{2} \in\right.$ $X\}$.

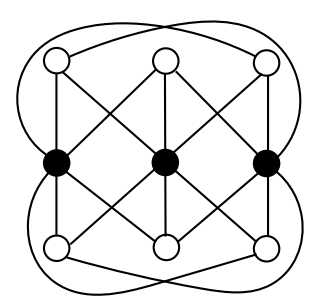

(a) $D$

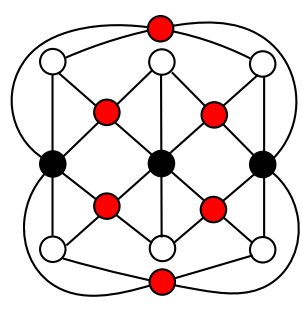

(b) $D^{\times}$

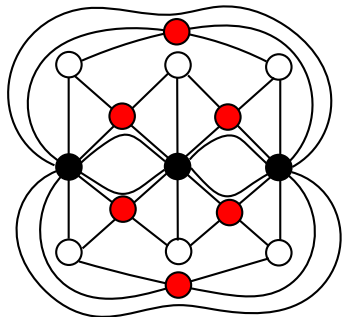

(c) $D_{W}^{\times}$

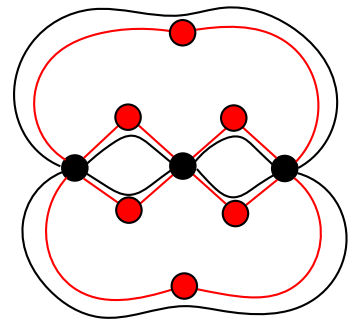

(d) $F$

Figure 2: $D, D^{\times}, D_{W}^{\times}$and $F$, where $D$ is a 1-planar drawing of $K_{3,6}$.

All vertices in $H$ are black and the edges in $H$ are also called black edges. Clearly, $W$ is an independent set in $F$ and each vertex in $W$ (i.e., a red vertex) is of degree 2 in $F$. The edges in $F$ incident with red vertices are called red edges. Thus, each edge in $F$ is either black or red, as shown in Figure $2(\mathrm{~d})$.

We have the following facts on $D^{\times}, F$ and $H$ :

(1) $D^{\times}, F$ and $H$ are simultaneously embedded in the plane;

(2) $F$ and $H$ are obviously loopless (A loop is an edge that starts and ends at the same vertex), but they are possibly disconnected;

(3) $w \rightarrow e_{w}$ is a bijection from $W$ to $E(H)$, where $w$ is a red vertex, and thus the number of crossings of $D$ equals to $|E(H)|$; and

(4) $e_{w} \rightarrow x_{1} w x_{2}$ is a bijection from $E(H)$ to the set of 2-paths in $F$ whose ends are black, where $w$ is a red vertex and $x_{1}$ and $x_{2}$ are the black vertices in $D^{\times}$adjacent to $w$.

Moreover we have the following propositions.

Proposition 6. Let $e_{w}$ be an edge of $H$ with ends $x_{1}$ and $x_{2}$ and $C$ be the 3-cycle of $F$ consisting of $e_{w}$ and its corresponding 2-path $P=x_{1} w x_{2}$, where $w$ is a red vertex (see Figure $3(a))$. Then int $(C)$ contains none of black vertices, red vertices and black edges in $F$; in this sense we also say that $\operatorname{int}(C)$ is "empty".

Proof. By the definition of $D_{W}^{\times}$, the proposition follows directly from the fact that the drawing of edge $e_{w}$ is the most near to one side of the 2-path $x_{1} w x_{2}$ in $D^{\times}$without crossings with edges in $D^{\times}$. 


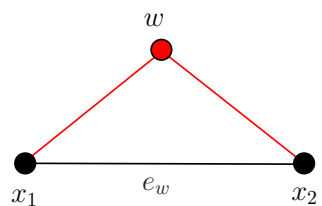

(a)

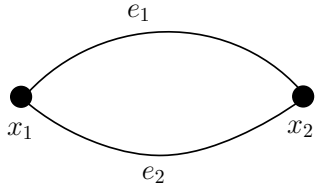

(b)

Figure 3: Some 3-cycles and 2-cycles in F.

Proposition 7. Assume that $H$ has no separating 2-cycles. If $C$ is a 2-cycle in $H$ that consists of two multiple edges $e_{1}$ and $e_{2}$ joining two black vertices $x_{1}$ and $x_{2}$ (see Figure 3 (b)), then either $\operatorname{int}(C)$ or ext $(C)$ contains neither black vertices nor red vertices.

Proof. As $H$ has no separating 2-cycles, either $\operatorname{int}(C)$ or $\operatorname{ext}(C)$ contains no black vertices. Assume that $\operatorname{int}(C)$ does not contain black vertices.

Suppose that $\operatorname{int}(C)$ contains red vertices. Then, $\operatorname{int}(C)$ contains white vertices of $D^{\times}$. Otherwise $C$ would be crossed. As $\operatorname{int}(C)$ does not contain black vertices, each white vertex in $\operatorname{int}(C)$ is of degree at most 2 in $D^{\times}$. Thus, we can redraw the edges of $D$ in $\operatorname{int}(C)$ such that these edges make no crossings, and then obtain a 1-planar drawing of $G$ with fewer crossings than $D$, contradicting to the choice of $D$. Hence $\operatorname{int}(C)$ does not contain red vertices and the conclusion holds.

Proposition 8. Assume that $H$ contains no separating 2-cycles. Then the edge multiplicity of $H$ is at most 2 .

Proof. Assume to contrary that $H$ has three multiple edges $e_{1}, e_{2}$ and $e_{3}$ which join the same pair of black vertices $x_{1}$ and $x_{2}$. Then these three edges divide the plane into three regions, denoted by $\alpha, \beta$ and $\gamma$, as shown in Figure 4 (a). By Proposition 7, at least two of these three regions contain neither red vertices nor black vertices, except on its boundary. We may assume $\alpha$ and $\gamma$ are such two regions.

Let $P=x_{1} w x_{2}$ be the 2-path of $F$ that corresponds to edge $e_{3}$, where $w$ is a red vertex. Thus, this path must be within region $\beta$, as shown in Figure 4 (b).

As $P$ is within region $\beta$, black edges $e_{1}$ and $e_{2}$ are in different sets $\operatorname{int}\left(e_{3} \cup P\right)$ and $\operatorname{ext}\left(e_{3} \cup P\right)$, a contradiction to Proposition 6. The proof is then completed.

An edge of $H$ is called a simple edge if it is not parallel to another edge in $H$ and a partnered edge otherwise. It follows from Proposition 8 that, if $H$ has no separating 2-cycles, then each partnered edge $e$ in $H$ is parallel to a unique partnered edge $e^{\prime}$ in $H$.

Let $C$ be a cycle and $P$ be a path in $H$ such that the end vertices of $P$ are the only vertices shared by $C$ and $P$. When we say that $P$ lies in $\operatorname{int}(C)($ resp. $\operatorname{ext}(C))$, it means that all edges and internal vertices of $P$ lie in $\operatorname{int}(C)($ resp. $\operatorname{ext}(C))$.

Proposition 9. Assume that $H$ has no separating 2-cycles. Let $C$ be a 3-cycle of $H$ consisting of black vertices $x_{1}, x_{2}$ and $x_{3}$, and $e$ be the edge on $C$ joining $x_{1}$ and $x_{3}$. 


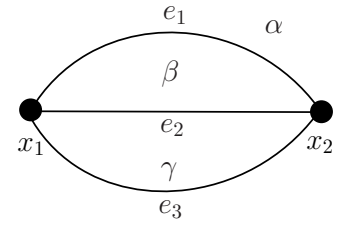

(a)

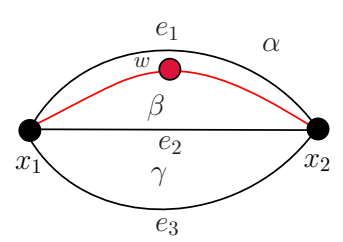

(b)

Figure 4: Possible three multiple edges.

Assume that $e^{\prime}$ is a partnered edge in $H$ which is parallel to e. If $P=x_{1} w x_{3}$ and $P^{\prime}=x_{1} w^{\prime} x_{3}$ are the 2-paths in $F$ corresponding to $e$ and $e^{\prime}$ respectively, then one of $P$ and $P^{\prime}$ lies in int $(C)$ and the other in $\operatorname{ext}(C)$.

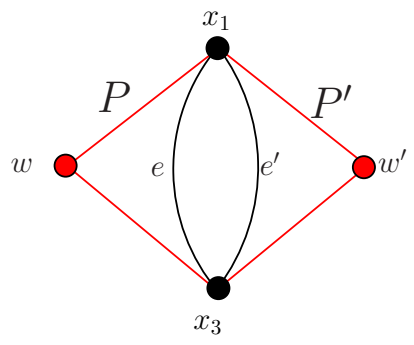

(a) $F\left[\left\{x_{1}, x_{3}, w, w^{\prime}\right\}\right]$

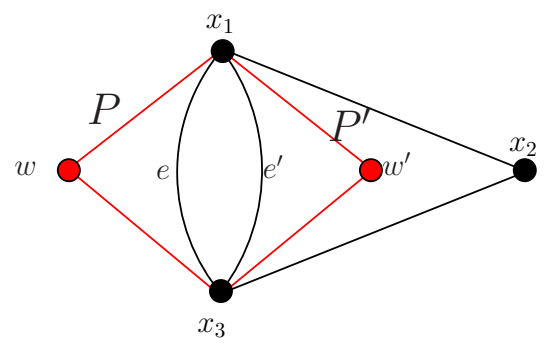

(b) $F\left[\left\{x_{1}, x_{2}, x_{3}, w, w^{\prime}\right\}\right]$

Figure 5: $x_{2}$ lies in $\operatorname{ext}\left(C_{2}\right)$, where $C_{2}$ is the cycle $x_{1} w x_{3} w^{\prime} x_{1}$.

Proof. Let $C_{0}$ denote the 2-cycle of $H$ consisting of edges $e$ and $e^{\prime}$. By Proposition 7, we may assume that $\operatorname{int}\left(C_{0}\right)$ contains neither black vertices nor red vertices. Thus, both $w$ and $w^{\prime}$ are in $\operatorname{ext}\left(C_{0}\right)$.

Let $C_{1}$ denote the 3 -cycle of $F$ consisting of edge $e$ and path $P$ and $C_{1}^{\prime}$ the 3 -cycle of $F$ consisting of edge $e^{\prime}$ and path $P^{\prime}$. By Proposition 6 , both $\operatorname{int}\left(C_{1}\right)$ and $\operatorname{int}\left(C_{1}^{\prime}\right)$ are empty. Thus, the subgraph $F\left[\left\{x_{1}, x_{3}, w, w^{\prime}\right\}\right]$ is as shown in Figure 5 (a).

As these three regions $\operatorname{int}\left(C_{0}\right), \operatorname{int}\left(C_{1}\right)$ and $\operatorname{int}\left(C_{1}^{\prime}\right)$ do not contain black vertices, $x_{2}$ must be in $\operatorname{ext}\left(C_{2}\right)$, where $C_{2}$ is the 4-cycle of $F$ consisting of paths $P=x_{1} w x_{3}$ and $P^{\prime}=x_{1} w^{\prime} x_{3}$. As $F$ is a plane graph, path $x_{1} x_{2} x_{3}$ must lie in $\operatorname{ext}\left(C_{2}\right)$, as shown in Figure 5(b). Hence the conclusion holds.

Proposition 10. Suppose that $H$ has no separating 2-cycles. For any 3-cycle $C$ in $H$, if int $(C)$ contains exactly $r$ red vertices, where $0 \leqslant r \leqslant 2$, then $C$ contains at least $3-r$ simple edges of $F$.

Proof. Let $e_{1}, e_{2}$ and $e_{3}$ be the three edges on $C$. Suppose that $e_{i}$ is not a simple edge of $H$, where $1 \leqslant i \leqslant 3$. Then $e_{i}$ is parallel to another partnered edge $e_{i}^{\prime}$ of $H$. Let $P_{i}$ 
and $P_{i}^{\prime}$ be 2-paths in $F$ which correspond to edges $e_{i}$ and $e_{i}^{\prime}$ respectively. Since $H$ has no separating 2-cycles, by Proposition $9, \operatorname{int}(C)$ contains a red vertex that is on $P_{i}$ or $P_{i}^{\prime}$.

The above conclusion implies that the number of red vertices in $\operatorname{int}(C)$ is not less than the number of partnered edges on $C$. Thus, the result holds.

\section{Upper bounds of $|E(G)|$ for some graphs $G$}

Let $G$ be a bipartite graph with partite sets $X$ and $Y$ and $\mathcal{O}$ be a disk on the plane up to a homeomorphism of the plane. If $D$ is a 1-planar drawing of $G$ that draws all vertices of $X$ on the boundary of $\mathcal{O}$ and all vertices of $Y$ and all edges of $G$ in the interior of $\mathcal{O}$, then we say that $D$ is a 1 -disk $\mathcal{O}_{X}$ drawing of $G$.

Lemma 11. Let $G$ be a bipartite graph with partite sets $X$ and $Y$, and let $D$ be a 1-disk $\mathcal{O}_{X}$ drawing of $G$ with the minimum number of crossings $k$. If $|X|=3$, then $k \in\{0,1,3\}$ and $|E(G)| \leqslant 2|Y|+\left\lfloor\frac{k+3}{2}\right\rfloor$, i.e.,

$$
|E(G)| \leqslant \begin{cases}2|Y|+1, & \text { if } k=0 \\ 2|Y|+2, & \text { if } k=1 \\ 2|Y|+3, & \text { if } k=3\end{cases}
$$

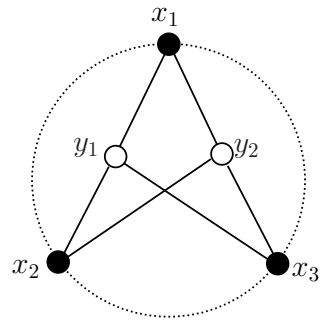

(a) $\left|Y_{3}\right|=2$

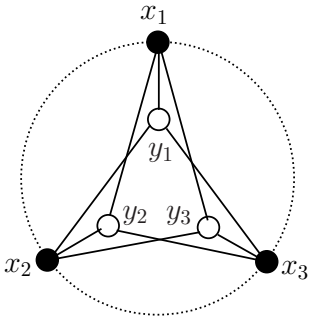

(b) $\left|Y_{3}\right|=3$

Figure 6: The 1-disk $\mathcal{O}_{X}$ drawing of $G$ for $Y=Y_{3}$ and $\left|Y_{3}\right| \in\{2,3\}$.

Proof. Assume that $|X|=3$. For any integer $i \geqslant 0$, let $Y_{i}$ be the set of vertices $y$ in $Y$ with $d_{G}(y)=i$. As $|X|=3$ and $Y$ is independent in $G, Y_{i}=\emptyset$ holds for all $i \geqslant 4$.

It can be checked easily that, for each vertex $y$ in $\in Y$, if $y \notin Y_{3}$, then $y$ is not incident with any crossed edge. Thus, $G-\bigcup_{i \leqslant 2} Y_{i}$ has exactly $k$ crossings, and it suffices to show that $\left|Y_{3}\right| \leqslant 3$ and

$$
k= \begin{cases}0, & \text { if }\left|Y_{3}\right| \leqslant 1 \\ 1, & \text { if }\left|Y_{3}\right|=2 \\ 3, & \text { if }\left|Y_{3}\right|=3\end{cases}
$$

The rest of the proof will be completed by showing the following claims.

Claim 12. $\left|Y_{3}\right| \leqslant 3$. 
Proof. Suppose that $\left|Y_{3}\right| \geqslant 4$. Then, there exists a bipartite 1-planar drawing $D^{\prime}$ isomorphic to $K_{3,2\left|Y_{3}\right|}$ obtained from $D\left[X \cup Y_{3}\right]$ by copying all vertices and edges in the interior of $\mathcal{O}_{X}$ to its exterior, implying that $K_{3,8}$ is 1-planar. It is a contradiction to the fact that $K_{3,7}$ is not 1-planar due to Czap and Hudák [3]. Thus, Claim 12 holds.

Claim 13. If $\left|Y_{3}\right| \leqslant 1$, then $G-\bigcup_{i \leqslant 2} Y_{i}$ has no crossings, i.e., $k=0$.

Proof. If $\left|Y_{3}\right| \leqslant 1$, the graph is a star, hence, in a simple drawing, there is no way to have a crossing.

Claim 14. For any two vertices $y_{1}, y_{2} \in Y_{3}$, some edge incident with $y_{1}$ crosses with some edge incident with $y_{2}$, as shown in Figure 6 (a).

Proof. If Claim 14 fails, then $G\left[X \cup\left\{y_{1}, y_{2}\right\}\right]$ is a plane graph and we can get a drawing of $K_{3,3}$ from $G\left[X \cup\left\{y_{1}, y_{2}\right\}\right]$ by adding a new vertex $y^{\prime}$ and three edges joining $y^{\prime}$ to all vertices in $X$ in the exterior of $\mathcal{O}_{X}$ without any crossing, implying that $K_{3,3}$ is planar, a contradiction.

Claim 15. $k=1$ when $\left|Y_{3}\right|=2$, and $k=3$ when $\left|Y_{3}\right|=3$.

Proof. By Claim 14, $k \geqslant\left(\begin{array}{c}\left|Y_{3}\right| \\ 2\end{array}\right)$. By the drawings in Figure $6, k \leqslant 1$ when $\left|Y_{3}\right| \leqslant 2$, and $k \leqslant 3$ when $\left|Y_{3}\right| \leqslant 3$. Thus, Claim 15 holds.

The result follows from Claims 12, 13 and 15.

Lemma 16. Let $G$ be a plane simple graph with $|V(G)| \geqslant 3$. If $G$ has exactly c components and $t(\geqslant 0)$ cellular 3 -faces, then $|E(G)| \leqslant 2|V(G)|-3-c+\frac{t}{2}$.

Proof. If $c=1$, since $G$ is simple, each face of $H$ is a cellular face and has size at least 3 . Then, in this case, the conclusion can be proved easily by applying the Euler's formula.

Now we assume that $c \geqslant 2$. We can obtain a simple and connected plane graph $G^{\prime}$ from $G$ by adding $c-1$ edges.

For every noncellular face $f$ of $G$ whose boundary consists of $\ell$ disjoint closed walks of $G$, we can add $\ell-1$ new edges without adding new vertices by appropriately drawing these new edges within $f$ so that $f$ is transformed into a cellular face of size at least 4 because $|V(G)| \geqslant 3$. Therefore, the resulting graph $G^{\prime}$ is a simple and connected plane, and all faces of $G^{\prime}$ are cellular.

Note that adding the $c-1$ new edges does not produce new cellular 3 -faces, and thus $G^{\prime}$ has exactly $t$ faces of size 3 . The conclusion for connected plane graphs implies that

$$
\left|E\left(G^{\prime}\right)\right| \leqslant 2\left|V\left(G^{\prime}\right)\right|-4+t / 2 .
$$

As $V\left(G^{\prime}\right)=V(G)$ and $\left|E\left(G^{\prime}\right)\right|=|E(G)|+c-1$, the above inequality implies that $|E(G)| \leqslant 2|V(G)|+t / 2-3-c$.

Lemma 17. Let $G$ be a simple and bipartite plane graph with $|V(G)| \geqslant 3$. If $G$ has exactly $c$ components and $t$ cellular faces whose boundaries are of length at least 6 , then $|E(G)| \leqslant 2|V(G)|-3-c-t$. 
Proof. If $G$ is connected (i.e. $c=1$ ), since $G$ is bipartite and simple, then each face of $G$ is a cellular face, and has the size at least 4 . Because $G$ has $t$ faces of size at least 6 , it follows from the Euler's formula that $|E(G)| \leqslant 2|V(G)|-4-t$.

Now assume that $c \geqslant 2$. We can obtain a simple and connected bipartite plane graph $G^{\prime}$ from $G$ by adding $c-1$ edges.

For every noncellular face $f$ of $G$ consisting of $\ell$ distinct closed walks, similar to the proof of Lemma 16, we can add $\ell-1$ new edges within this noncellular face so that $f$ is transformed into a cellular face. We can ensure that those new added edges join the vertices in different partite sets of $G$. Hence the resulting plane graph $G^{\prime}$ is simple, bipartite and connected. Clearly, all faces of $G^{\prime}$ are cellular, and $G^{\prime}$ has at least $t$ faces whose boundaries are of length at least 6 . The conclusion for bipartite and connected plane graphs implies that

$$
\left|E\left(G^{\prime}\right)\right| \leqslant 2\left|V\left(G^{\prime}\right)\right|-4-t .
$$

As $V\left(G^{\prime}\right)=V(G)$ and $\left|E\left(G^{\prime}\right)\right|=|E(G)|+c-1$, the above inequality implies that $|E(G)| \leqslant 2|V(G)|-3-c-t$.

Remark 18. Lemmas 16 and 17 can be strengthened when $G$ contains isolated vertices. Let $V_{\geqslant 1}(G)$ be the set of non-isolated vertices in $G$. Then, under the condition $\left|V_{\geqslant 1}(G)\right| \geqslant 3$, the conclusions of both Lemmas 16 and 17 still hold after $|V(G)|$ is replaced by $\left|V_{\geqslant 1}(G)\right|$.

\section{Proof of Theorem 4}

The whole section contributes to the proof of Theorem 4 .

Proof of Theorem 4. Suppose that Theorem 4 fails and $\chi$ is the minimum integer with $\chi \geqslant 2$ such that for some bipartite 1-planar graph $G$ with partite sets $X$ and $Y$, where $\chi=|X| \leqslant|Y|,|E(G)|>2|V(G)|+4|X|-12$ holds.

We will prove the following claims to show that this assumption leads to a contradiction.

Claim 19. $\chi \geqslant 4$.

Proof. Let $G$ be any bipartite 1-planar graph with bipartitions $X$ and $Y$, where $2 \leqslant|X| \leqslant$ $|Y|$. If $|X|=2$, obviously,

$$
|E(G)| \leqslant 2|Y|=2(2+|Y|)+4 \times 2-12=2|V(G)|+4|X|-12 .
$$

Now assume that $|X|=3$. Let $Y_{i}$ be the set of vertices $y$ in $Y$ with $d_{G}(y)=i$. Then $|E(G)| \leqslant 2|Y|+\left|Y_{3}\right|$. Since the complete bipartite graph $K_{3,7}$ is not 1-planar (see [3]), we have $\left|Y_{3}\right| \leqslant 6$, implying that $|E(G)| \leqslant 2|Y|+6$. As $x=3$, we have

$$
2|V(G)|+4|X|-12=6|X|+2|Y|-12=2|Y|+6 .
$$

Thus, $|E(G)| \leqslant 2|V(G)|+4|X|-12$.

By the assumption of $\chi$, we have $\chi \geqslant 4$. 
In the following, we assume that $G$ is a bipartite 1-planar graph with bipartitions $X$ and $Y$, where $\chi=|X| \leqslant|Y|$, such that

$$
|E(G)|>2|V(G)|+4|X|-12 .
$$

Let $D$ be a 1-planar drawing of $G$ with the minimum number of crossings and $W$ be the set of its crossings. Introduced in Section $3, D_{W}^{\times}$is a plane graph extended from $D^{\times}$, and $F$ and $H$ are the subgraphs $D_{W}^{\times}[X \cup W]$ and $D_{W}^{\times}[X]$ of $D_{W}^{\times}$respectively. All vertices in $X$ are black vertices, all vertices in $Y$ are white vertices and all vertices in $W$ are red vertices.

We are now going to prove the following claim.

Claim 20. $H$ has no separating 2-cycles.

Proof. Assume to the contrary that $H$ has a separating 2-cycle $C$ consisting of two parallel edges $e_{1}$ and $e_{2}$ joining black vertices $x_{1}$ and $x_{2}$ (see Figure $3(\mathrm{~b})$ ), such that both $\operatorname{int}(C)$ and $\operatorname{ext}(C)$ contain black vertices.

Let $G_{1}=G \bigcap I N T(C)$ and $G_{2}=G \bigcap E X T(C)$. Obviously, both $G_{1}$ and $G_{2}$ are bipartite 1-planar subgraphs of $G$. Moreover, we can see that $G_{1} \cup G_{2}=G$, and $G_{1} \cap G_{2}=$ $\left\{x_{1}, x_{2}\right\}$.

For $i=1,2, G_{i}$ has a bipartition $X_{i}$ and $Y_{i}$, where $X_{i}=X \cap V\left(G_{i}\right)$ and $Y_{i}=Y \cap V\left(G_{i}\right)$. Clearly, $\left|X_{1}\right|+\left|X_{2}\right|=|X|+2=\chi+2$ and $\left|Y_{1}\right|+\left|Y_{2}\right|=|Y|$.

Since $C$ is a separating cycle of $H$, both $\operatorname{int}(C)$ and $\operatorname{ext}(C)$ contain black vertices, implying that $\left|X_{i}\right| \geqslant 3$ for both $i=1,2$. As $\left|X_{1}\right|+\left|X_{2}\right|=|X|+2$, we have $\left|X_{i}\right|<|X|=\chi$ and so $\min \left\{\left|X_{i}\right|,\left|Y_{i}\right|\right\} \leqslant\left|X_{i}\right|<\chi$.

For $i=1,2$, if $\left|Y_{i}\right| \leqslant 1$, then $\left|E\left(G_{i}\right)\right| \leqslant\left|Y_{i}\right| \cdot\left|X_{i}\right|$ and $\left|E\left(G_{i}\right)\right| \leqslant 2\left|V\left(G_{i}\right)\right|+4\left|X_{i}\right|-12$ holds; if $\left|Y_{i}\right| \geqslant 2$, then $2 \leqslant \min \left\{\left|X_{i}\right|,\left|Y_{i}\right|\right\} \leqslant\left|X_{i}\right|<\chi$ and the assumption on $\chi$ implies that the conclusion of Theorem 4 holds for $G_{i}$, i.e.,

$$
\left|E\left(G_{i}\right)\right| \leqslant 2\left|V\left(G_{i}\right)\right|+4 \min \left\{\left|X_{i}\right|,\left|Y_{i}\right|\right\}-12 \leqslant 2\left|V\left(G_{i}\right)\right|+4\left|X_{i}\right|-12 .
$$

Thus, by (2),

$$
\begin{aligned}
|E(G)| & =\left|E\left(G_{1}\right)\right|+\left|E\left(G_{2}\right)\right| \\
& \leqslant 2\left(\left|V\left(G_{1}\right)\right|+\left|V\left(G_{2}\right)\right|\right)+4\left(\left|X_{1}\right|+\left|X_{2}\right|\right)-24 \\
& =2(|V(G)|+2)+4(|X|+2)-24 \\
& =2|V(G)|+4|X|-12,
\end{aligned}
$$

which contradicts to the assumption in (1).

Hence Claim 20 holds.

It is known from Proposition 8 that the edge multiplicity of each edge in $H$ is at most 2. Then, there exists a subset $A$ of $E(H)$ such that both $H\langle A\rangle$ and $H-A$ are simple graphs and each edge in $A$ is parallel to some edge in $E(H)-A$, where $H\langle A\rangle$ is the spanning subgraph of $H$ with edge set $A$ and $H-A$ is the graph obtained from $H$ by 
removing all edges in $A$. Clearly, $|A|$ is the number of pairs of edges $e$ and $e^{\prime}$ in $H$ which are parallel.

Let $H^{\prime}$ denote $H-A$. Obviously, $|A| \leqslant\left|E\left(H^{\prime}\right)\right|$, and

$$
|E(H)|=\left|E\left(H^{\prime}\right)\right|+|A| \text {. }
$$

Claim 21. $H^{\prime}$ contains at least one cellular 3-face.

Proof. Suppose that $H^{\prime}$ has no cellular 3-faces. As $H^{\prime}$ is a simple plane graph, by Lemma $16,\left|E\left(H^{\prime}\right)\right| \leqslant 2\left|V\left(H^{\prime}\right)\right|-4=2|X|-4$. Because $|A| \leqslant\left|E\left(H^{\prime}\right)\right|$, it follows from (4) that $|E(H)| \leqslant 2\left|E\left(H^{\prime}\right)\right| \leqslant 4|X|-8$.

Since each edge of $H$ is in 1-1 correspondence with a crossing of a drawing $D$ of $G$, we can obtain a simple bipartite plane graph (possibly disconnected), denoted by $G^{\prime}$, by removing $|E(H)|$ edges from $G$ each of which is a crossed edge of $G$. By Lemma 17, $\left|E\left(G^{\prime}\right)\right| \leqslant 2\left|V\left(G^{\prime}\right)\right|-4=2|V(G)|-4$. Therefore,

$$
|E(G)|=\left|E\left(G^{\prime}\right)\right|+|E(H)| \leqslant\left|E\left(G^{\prime}\right)\right|+4|X|-8=2|V(G)|+4|X|-12,
$$

a contradiction to the assumption in (1).

Hence Claim 21 holds.

Now we assume that $H^{\prime}$ has exactly $t$ cellular 3 -faces, where $t \geqslant 1$. Let $\mathscr{T}\left(H^{\prime}\right)$ denote the set of cellular 3 -faces in $H^{\prime}$. So $t=\left|\mathscr{T}\left(H^{\prime}\right)\right|$.

For each $\Delta \in \mathscr{T}\left(H^{\prime}\right)$, for convenience we also use " $\Delta$ " to represent the 3 -cycle corresponding the boundary of $\Delta$ if there is no confusions in the context. Let $G_{\Delta}=$ $G \bigcap \operatorname{INT}(\Delta)$. Since $\Delta$ is a cellular 3 -face of $H^{\prime}$, there are no black vertices lying in $\operatorname{int}(\Delta)$, and thus $G_{\Delta}$ is a bipartite with exactly three black vertices, which lie on the boundary of the face $\Delta$.

Let $\Delta \in \mathscr{T}\left(H^{\prime}\right)$. Since $D$ is a 1-planar drawing of $G$ with minimal number of crossings, the induced subdrawing of $D$ of $G_{\Delta}$ is a 1-disk $\mathcal{O}_{X_{\Delta}}$ drawing of $G_{\Delta}$ with the minimum number of crossings, where $X_{\Delta}$ is the set of three black vertices on the boundary of $\Delta$. Otherwise, we redraw the edges of $G$ lying in the interior of $\Delta$, and obtain a 1-planar drawing of $G$ with fewer crossings than $D$, contradicting to the choice of $D$. By Lemma 11 , the number of crossings of $D$ in $\operatorname{int}(\Delta)$ is a number in the set $\{0,1,3\}$.

For any $j \in\{0,1,3\}$, let $\mathscr{T}^{(j)}\left(H^{\prime}\right)$ be the set of members $\Delta$ in $\mathscr{T}\left(H^{\prime}\right)$ such that $\operatorname{int}(\Delta)$ contains exactly $j$ crossings of $D$. Assume that $\mathscr{T}^{(j)}\left(H^{\prime}\right)=\left\{\Delta_{i}^{(j)}: 1 \leqslant i \leqslant t_{j}\right\}$, where $t_{j}=\left|\mathscr{T}^{(j)}\left(H^{\prime}\right)\right|$. Thus, $t_{0}+t_{1}+t_{3}=t$.

For each $\Delta_{i}^{(j)} \in \mathscr{T}^{(j)}\left(H^{\prime}\right)$, let $e_{i}^{(j)}$ be the number of the edges of the graph $G_{\Delta_{i}^{(j)}}$ and $y_{i}^{(j)}$ be the number of white vertices in $\operatorname{int}\left(\Delta_{i}^{(j)}\right)$.

Claim 22. $\sum_{j \in\{0,1,3\}} \sum_{i=1}^{t_{j}} e_{i}^{(j)} \leqslant 2 \sum_{j \in\{0,1,3\}} \sum_{i=1}^{t_{j}} y_{i}^{(j)}+\left(t_{0}+2 t_{1}+3 t_{3}\right)$.

Proof. By Lemma 11, $e_{i}^{(j)} \leqslant 2 y_{i}^{(j)}+\left\lfloor\frac{j+3}{2}\right\rfloor$ holds for any $j \in\{0,1,3\}$ and $1 \leqslant i \leqslant t_{j}$. Thus, Claim 22 holds. 
Claim 23. $|E(H)| \leqslant 4|X|-8+t-\left(3 t_{0}+2 t_{1}\right) / 2$.

Proof. Since $H^{\prime}$ is a simple plane graph with exactly $t \geqslant 1$ cellular 3 -faces and $\left|V\left(H^{\prime}\right)\right|=$ $|X|=\chi \geqslant 4$, by Lemma 16,

$$
\left|E\left(H^{\prime}\right)\right| \leqslant 2\left|V\left(H^{\prime}\right)\right|-4+\frac{t}{2}=2|X|-4+\frac{t}{2} .
$$

On the other hand, by Proposition 10 , for any $j \in\{0,1,3\}$ and $1 \leqslant i \leqslant t_{j}$, at least $3-j$ edges on the boundary of $\Delta_{i}^{(j)}$ are simple edges, implying that at least $3-j$ edges on the boundary of $\Delta_{i}^{(j)}$ are in $H^{\prime}$. Because each simple edge of $H^{\prime}$ belongs to the boundaries of at most two different faces of $H^{\prime}$, it follows that $\left|E\left(H^{\prime}\right)\right| \geqslant\left(3 t_{0}+2 t_{1}\right) / 2$. Then,

$$
|A| \leqslant\left|E\left(H^{\prime}\right)\right|-\left(3 t_{0}+2 t_{1}\right) / 2
$$

and therefore, by (4) and (5),

$$
|E(H)|=\left|E\left(H^{\prime}\right)\right|+|A| \leqslant 4|X|-8+t-\left(3 t_{0}+2 t_{1}\right) / 2 .
$$

Thus, Claim 23 holds.

Let $D^{\prime}$ denote the drawing obtained from $D$ by deleting all white vertices and edges of $D$ that lie in the interiors of all cellular 3-faces $\Delta_{i}^{(j)}$ of $H^{\prime}$, where $j \in\{0,1,3\}$ and $1 \leqslant i \leqslant t_{j}$, and let $G^{\prime}$ denote the graph represented by $D^{\prime}$.

We see that the graph $G^{\prime}$ is a bipartite 1-planar graph with a bipartition $X$ and $Y^{\prime}=Y \cap V\left(G^{\prime}\right)$, where $\left|Y^{\prime}\right|=|Y|-\sum_{j \in\{0,1,3\}} \sum_{i=1}^{t_{j}} y_{i}^{(j)}$. Thus,

$$
\left|V\left(G^{\prime}\right)\right|=|V(G)|-\sum_{j \in\{0,1,3\}} \sum_{i=1}^{t_{j}} y_{i}^{(j)}
$$

and

$$
\left|E\left(G^{\prime}\right)\right|=|E(G)|-\sum_{j \in\{0,1,3\}} \sum_{i=1}^{t_{j}} e_{i}^{(j)} .
$$

As the number of crossings of $D$ equals to $|E(H)|$ and $D^{\prime}$ has no crossings lying in the interior of any cellular 3-face of $H^{\prime}, D^{\prime}$ has exactly $|E(H)|-\left(t_{1}+3 t_{3}\right)$ crossings.

For each crossing of $D^{\prime}$, we remove exactly one crossed edge from $G^{\prime}$ and obtain a bipartite plane graph $G^{*}$. Thus, $\left|E\left(G^{*}\right)\right|=\left|E\left(G^{\prime}\right)\right|-\left(|E(H)|-\left(t_{1}+3 t_{3}\right)\right)$. Then, (8) implies that

$$
\left|E\left(G^{*}\right)\right|=\left(|E(G)|-\sum_{j \in\{0,1,3\}} \sum_{i=1}^{t_{j}} e_{i}^{(j)}\right)-|E(H)|+\left(t_{1}+3 t_{3}\right) .
$$


Clearly, by (7),

$$
|V(G)|=\left|V\left(G^{\prime}\right)\right|+\sum_{j \in\{0,1,3\}} \sum_{i=1}^{t_{j}} y_{i}^{(j)}=\left|V\left(G^{*}\right)\right|+\sum_{j \in\{0,1,3\}} \sum_{i=1}^{t_{j}} y_{i}^{(j)} .
$$

Now, we shall obtain an upper bound of $\left|E\left(G^{*}\right)\right|$ in terms of $\left|V\left(G^{*}\right)\right|$ by constructing a bipartite plane graph with at least $t$ cellular 6 -faces.

Claim 24. $\left|E\left(G^{*}\right)\right| \leqslant 2\left|V\left(G^{*}\right)\right|-4-t$.

Proof. Note that the simple and bipartite plane graph $G^{*}$ is obtained from $G$ by removing all white vertices and edges of $G$ lying in the interiors of all cellular 3-faces of $H^{\prime}$ and, for each crossing of $D$ not lying in any cellular 3-face of $H^{\prime}$, removing exactly one edge of $G$ involved in this crossing.

Now let $G^{* *}$ denote the graph obtained from $G^{*}$ by adding all black edges in $H^{\prime}$ which belong to the boundary of cellular 3-faces of $H^{\prime}$ and then subdividing each of these added edges by exactly one vertex. Let $m$ be the number of edges in $H^{\prime}$ that belong to the boundaries of cellular 3 -faces of $H^{\prime}$. Then

$$
\left|V\left(G^{* *}\right)\right|=\left|V\left(G^{*}\right)\right|+m \text { and }\left|E\left(G^{* *}\right)\right|=\left|E\left(G^{*}\right)\right|+2 m .
$$

Because the edges of $H$ (and thus $H^{\prime}$ ) are not crossed with the edges of $G$ (and thus $\left.G^{*}\right)$, we observe that $G^{* *}$ is also a simple and bipartite plane graph and has at least $t$ cellular 6-faces. Applying Lemma 17 to $G^{* *}$ yields that

$$
\left|E\left(G^{* *}\right)\right| \leqslant 2\left|V\left(G^{* *}\right)\right|-4-t .
$$

Then, (11) implies that $\left|E\left(G^{*}\right)\right| \leqslant 2\left|V\left(G^{*}\right)\right|-4-t$. This proves the claim.

Claim 25. $|E(G)| \leqslant 2|V(G)|+4|X|-12-t_{0} / 2$.

Proof. By (9), we have

$$
|E(G)|=\left|E\left(G^{*}\right)\right|+|E(H)|+\sum_{j \in\{0,1,3\}} \sum_{i=1}^{t_{j}} e_{i}^{(j)}-\left(t_{1}+3 t_{3}\right) .
$$

Then, by Claims 22, 23 and 24,

$$
\begin{aligned}
|E(G)| \leqslant & \left(2\left|V\left(G^{*}\right)\right|-4-t\right)+\left(4|X|-8+t-\left(3 t_{0}+2 t_{1}\right) / 2\right) \\
& +\left(2 \sum_{j \in\{0,1,3\}} \sum_{i=1}^{t_{j}} y_{i}^{(j)}+\left(t_{0}+2 t_{1}+3 t_{3}\right)\right)-\left(t_{1}+3 t_{3}\right) \\
= & 2|V(G)|+4|X|-12-t_{0} / 2 \\
\leqslant & 2|V(G)|+4|X|-12 .
\end{aligned}
$$

Clearly, Claim 25 contradicts the assumption in (1). Hence Theorem 4 holds. 


\section{Remarks}

For any $x \geqslant 3$ and $y \geqslant 6 x-12$, Czap, Przybyło and Škrabul'áková [5, Lemma 4] constructed a bipartite 1-planar graph $G$ with partite sets $X$ and $Y$ such that $|E(G)|=$ $2|V(G)|+4|X|-12$. Notice that the 1-planar drawing $D$ of this graph $G$ given in [5] has the following property:

$\left(^{*}\right)$ each vertex in $X$ is incident with crossed edges in $D$.

The proof of Theorem 4 also yields that, if $|E(G)|=2|V(G)|+4|X|-12$ holds for a bipartite 1-planar graph $G$ with partite sets $X$ and $Y$, where $4 \leqslant|X| \leqslant|Y|$, and $D$ is a 1-planar drawing of $G$ with the minimum number of crossings, then the graph $H^{\prime}$ introduced in Section 3 does not have isolated vertices, i.e., property $\left(^{*}\right)$ above holds.

Based on the above observations, we propose the following problem.

Problem 26. For any bipartite 1-planar graph $G$ with partite sets $X$ and $Y$, where $4 \leqslant|X| \leqslant|Y|$, if $|E(G)|=2|V(G)|+4|X|-12$, does property (*) hold for every 1-planar drawing $D$ of $G$ with the minimum number of crossings?

From Claims 21 and 23 in the proof of Theorem 4, we can see that if $H^{\prime}$ does not have separating 2-cycles and $\left|X_{>0}\right| \geqslant 3$, where $X_{>0}$ is the set of non-isolated vertices in $H^{\prime}$ (i.e., the set of vertices in $X$ which are incident with crossed edges of $D$ ), then $|E(G)| \leqslant 2|V(G)|+4\left|X_{>0}\right|-12$ holds.

Problem 27. Let $G$ be a bipartite 1-planar graph with partite sets $X$ and $Y$, where $4 \leqslant|X| \leqslant|Y|$. If $D$ is a 1-planar drawing of $G$ with the minimum number of crossings and $\left|X_{>0}\right| \geqslant 3$, where $X_{>0}$ is the set of vertices in $X$ which are incident with crossed edges of $D$, does $|E(G)| \leqslant 2|V(G)|+4\left|X_{>0}\right|-12$ hold?

Theorem 4 shows that $|E(G)| \leqslant 2|V(G)|+4 x-12$ holds for any bipartite 1-planar graph $G$ with bipartite sets of sizes $x$ and $y$, where $2 \leqslant x \leqslant y$. For any $x \geqslant 3$ and $y \geqslant 6 x-12$, Czap, Przybyło and Škrabul'áková [5] constructed a bipartite 1-planar graph $G$ with bipartite sets of sizes $x$ and $y$ and $|E(G)|=2|V(G)|+4 x-12$. Notice that these graphs constructed in [5] have minimum degree 3. By Theorem 1, any bipartite 1-planar graph of $n$ vertices has at most $3 n-8$ edges, implying that its minimum degree is at most 5. We wonder if the result in Theorem 4 can be improved for bipartite 1-planar graphs with higher minimum degrees or connectivity.

Problem 28. Let $4 \leqslant t \leqslant 5$ and $G$ be any bipartite 1 -planar graph with partite sets $X$ and $Y$, where $t \leqslant|X| \leqslant|Y|$. If $G$ is $t$-connected (or $\delta(G)=t$ ), does $|E(G)| \leqslant$ $2|V(G)|+f(t)|X|+c$ hold for some $f(t)<4$ ?

Let $t \geqslant 2$. A drawing of a graph is t-planar if each of its edges is crossed at most $t$ times. If a graph has a $t$-planar drawing, then it is t-planar. Does Theorem 4 have an analogous result for bipartite 2-planar graphs?

Problem 29. Let $G$ be a bipartite 2-planar graph with partite sets $X$ and $Y$, where $2 \leqslant|X| \leqslant|Y|$. Determine constants $a, b$ and $c$ such that $|E(G)| \leqslant a|V(G)|+b|X|+c$. 
Lemma 11 gives an upper bound for the size of a bipartite graph $G$ with partite sets $X$ and $Y$, where $|X|=3$, which has a 1-disk $\mathcal{O}_{X}$ drawing. Can this result be generalized for such a bipartite graph without the condition that $|X|=3$ ?

Problem 30. Let $G$ be a bipartite graph with partite sets $X$ and $Y$ which has a 1-disk $\mathcal{O}_{X}$ drawing. Is it true that $|E(G)| \leqslant 2|Y|+5|X| / 3-2$ ?

A straight line embedding of a graph is a graph embedding in which all edges are drawn as straight line segments. It was proved by Thomassen [12] that every 1-planar graph admits a straight-line drawing if and only if it does not contain a B-configuration or a W-configuration, as shown in Figure 7.

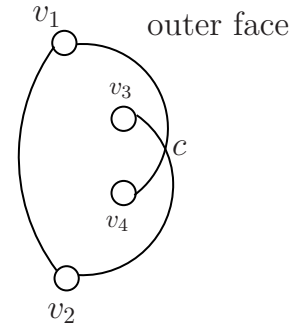

(a) B-configuration

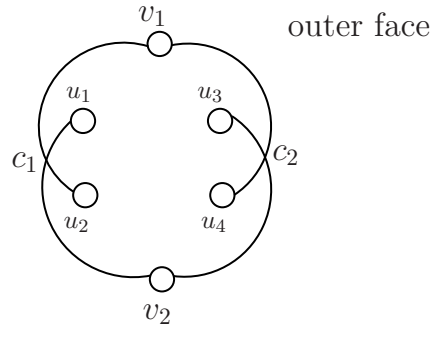

(b) W-configuration

Figure 7: (a) A B-configuration consists of an edge $v_{1} v_{2}$ and two edges $v_{1} v_{4}$ and $v_{2} v_{3}$ which cross at point $c$ such that $v_{3}$ and $v_{4}$ lie in the interior of the triangle formed by $v_{1}, v_{2}$ and $c$. (b) A W-configuration consists of edges $v_{1} u_{2}, v_{1} u_{4}, v_{2} u_{1}, v_{2} u_{3}$, where $v_{1} u_{2}$ and $v_{2} u_{1}$ cross at point $c_{1}$ and $v_{1} u_{4}$ and $v_{2} u_{3}$ cross at point $c_{2}$, such that $u_{1}, u_{2}, u_{3}$ and $u_{4}$ lie in the interior of the quadrangle formed by $c_{1}, c_{2}, v_{1}$ and $v_{2}$. It is possible that $u_{1}=u_{4}$ and $u_{2}=u_{3}$.

Theorem 4 determines the maximum size of a bipartite 1-planar graph. We wonder whether such a graph is straight-line drawable.

Problem 31. Is every maximally-dense bipartite 1-planar graph straight-line drawable?

By Thomassen's characterization, to show that a maximally-dense bipartite 1-planar graph $G$ is straight-line drawable, it is sufficient to show that $G$ has a 1-planar embedding which does not contain any B-configuration or W-configuration.

\section{Acknowledgements}

The authors would like to thank the anonymous referees for many helpful comments and suggestions.

\section{References}

[1] J. A. Bondy and U. S. R. Murty. Graph Theory, Grad. Texts in Math., vol.244, Springer, New York, 2008. 
[2] H. Bodendiek, R. Schumacher, and K. Wagner. Über 1-optimale Graphen. Math. Nachr., 117:323-339, 1984.

[3] J. Czap and D. Hudák. 1-planarity of complete multipartite graphs. Discrete Appl. Math., 160: 505-512, 2012.

[4] J. Czap and D. Hudák and T. Madaras. Joins of 1-planar graphs. Acta Mathematica Sinica, English Series, 30(11):1867-1876, 2014.

[5] J. Czap, J. Przybyło and E. Škrabul'áková. On an extremal problem in the class of bipartite 1-planar graphs. Discuss. Math., Graph Theory, 36:141-151, 2016.

[6] I. Fabrici and T. Madaras. The structure of 1-planar graphs. Discrete Math., 307:854865, 2007.

[7] D. V. Karpov. Upper bound on the number of edges of an almost planar bipartite graph. J. Math. Sci., 196:737-746, 2014.

[8] S. G. Kobourov, G. Liotta and F. Montecchiani. An annotated bibliography on 1-planarity. Comput. Sci. Rev., 25:49-67, 2017.

[9] J. Pach and G. Tóth. Graphs drawn with few crossings per edge. Combinatorica, 17(3):427-439, 1997.

[10] G. Ringel. Ein Sechsfarbenproblem auf der Kugel. Abh. Math. Sem. Univ. Hamburg, 29:107-117, 1965.

[11] E. Sopena. Personal communication, Seventh Cracow Conference in Graph Theory. Rytro, 2014.

[12] C. Thomassen. Rectilinear drawings of graphs. J. Graph Theory, 10(3):335-341, 1988.

[13] X. Zhang. Drawing complete multipartite graphs on the plane with restrictions on crossings. Acta Mathematica Sinica, English Series, 30(12): 2045-2053, 2014. 